\title{
Chaos in a topologically transitive semi-flow
}

\author{
Risong Lia,*, Tianxiu Lü \\ ${ }^{a}$ School of Mathematic and Computer Science, Guangdong Ocean University, Zhanjiang, Guangdong, 524025, P. R. China. \\ ${ }^{b}$ Department of Mathematics, Sichuan University of Science and Engineering, Zigong, Sichuan, 643000, P. R. China.
}

Communicated by D. Baleanu

\begin{abstract}
In this paper, we study the chaotic phenomena in a topologically transitive, continuous semi-flow, and show that the erratic time dependence of orbits in such a semi-flow is more complicated than the one described by Li-Yorke chaos. Also, we generalize the notion of sensitive dependence on initial conditions for semi-flows and explore the chaotic phenomena for topologically transitive, continuous semi-flows with the generalized sensitivity property. Our results extend the existing results to semi-flows. (C)2017 All rights reserved.
\end{abstract}

Keywords: Chaos, topological transitivity, sensitive dependence.

2010 MSC: 54H20, 37A05, 28D05, 37A25.

\section{Introduction}

A transitive system with sensitive dependence on initial conditions (briefly, sensitivity) was considered as a chaotic system by Ruelle and Takens [24]. This problem has gained much attention recently (see $[1,2,4,7,14,15,21,25,26,30-36])$. A system is Li-Yorke chaotic if there exists an uncountable scrambled set in its domain [16]. A transitive system is Devaney chaotic if it is a Ruelle-Takens chaotic system with a dense set of periodic points [7]. However, it is known that a transitive system with a dense set of periodic points is sensitive (see [4]). Huang and Ye [13] studied the transitive system with a fixed point, and they proved that such a system is Li-Yorke chaotic. For a transitive system with sensitivity, in [20] the author gave a scrambled set which is crueller than that in the original sense of $\mathrm{Li}$ and Yorke. Xiong et al. (see $[38,39]$ described and studied the chaotic phenomena caused by some maps by using the methods which are different from Li and Yorke's. In [37] the author followed the ideas and approaches in $[38,39]$ to explore, discover and characterize the chaotic phenomena in a transitive system, and he showed that to characterize the chaotic phenomena it is not adequate only by Li-Yorke chaos. Also, he gave two generic properties of the power systems of a transitive system and generalized the concept of sensitivity. Moreover, he discussed the chaotic phenomena for topologically transitive maps with this kind of sensitivity property and proved that another generic property of the power systems which is relative

\footnotetext{
*Corresponding author

Email addresses: gdoulrs@163.com (Risong Li), lubeeltx@163.com (Tianxiu Lu)
} 
to such a sensitivity property. For transitive maps on topological spaces, Bilokopytov and Kolyada [6] studied the problem of existence of some nonequivalent definitions of topological transitivity. So, this is a classical problem of the topological dynamics. Particularly, they used the fact that all available definitions of this kind imply a condition imposed on the dynamical system and obtained the complete classification of these dynamical systems, which is an important and useful result. In [40], by using the properties of weakly almost periodic points of a dynamical system $(X, f)$ having at least two points Yin and Zhou showed that, if the measure center of $f$ is the whole space, then there are seven equivalent conditions for the map $f$ to be ergodic mixing. Also, they gave an important application. There exist lots of chaotic phenomena in the real world. Moreover, there are a lot of important and possible applications of chaotic maps. In [27], by a nonlinear coupling method the authors studied master-slave synchronization for the fractional difference equation. By the numerical simulation they showed that the designed synchronization method can effectively synchronize the fractional logistic map, and that the Caputo-like delta derivative is adopted as the difference operator. In [28], the authors proposed a discrete fractional logistic map in the left Caputo discrete delta's sense and showed that the new model holds discrete memory. Also, they gave the bifurcation diagrams and numerically illustrated the chaotic behaviors. This means that there are important applications of this map. In [29], the authors presented fractional logistic map and fractional Lorenz maps of Riemann-Liouville type and studied the general chaotic behaviors of these maps in comparison with the Caputo one. Furthermore, they designed chaos synchronization according to the stability results. By the obtained numerical results we can see the method's effectiveness and fractional chaotic map's potential role for secure communication. In [12], the fractional chaotic map was applied in physics and engineering to properly treat some real-world phenomena. A shuffling method was presented based on the fractional logistic map. The fractional difference order plays a key pole in this problem. An image encryption scheme was designed by using the XOR operation and the security analysis was obtained. The obtained results show that the fractional difference order can make the encryption scheme highly secure. There have been a lot of results for dynamical properties of semi-flows. In [11], the authors studied the dynamical properties of continuous semi-flows with topological transitivity and obtained several interesting results. In [5], the authors proved that for a nonsingular (fixed point free) $C^{1}$ flow on a smooth closed 3-dimensional manifold $M$ with $\mathrm{H}_{2}(M)=0$, if this flow has a dense orbit then there exists an open dense set $\mathrm{N} \subset \mathrm{M}$ such that any knotted periodic orbit which intersects $\mathrm{N}$ is a nontrivial prime knot. In [8] Gu introduced the asymptotic average-shadowing property for flows and studied the relationships between this property and transitivity for flows. It was showed that a flow on a compact metric space is chain transitive if it has positively (or negatively) asymptotic average-shadowing property and a positively (resp. negatively) Lyapunov stable flow is positively (resp. negatively) topologically transitive provided it has positively (resp. negatively) asymptotic average-shadowing property. Furthermore, two conditions for which a flow is a minimal flow are given. Therefore, research on dynamical properties of continuous semi-flows is very interesting. Furthermore, from [3, 9, 10, 14, 15, 17-19] research on dynamical properties of continuous semi-flows is very difficult.

In this article, we consider the chaotic phenomena in a topologically transitive, continuous semi-flow on a complete metric space, and obtain that the erratic time dependence of orbits in such a semi-flow is more complicated than Li-Yorke chaos. Also, we generalize the notion of sensitive dependence on initial conditions (briefly, sensitivity) for semi-flows and investigate the chaotic phenomena for topologically transitive semi-flows with such a sensitivity property. Our results extend some existing results to semiflows.

The rest of this paper is organized as follows. In Section 2, we recall some basic concepts and notations. Main results are established in Section 3.

\section{Preliminaries}

Let $\mathbb{N}=\{1,2, \cdots\}$ and $\mathbf{R}^{+}=[0,+\infty)$. For the related notations and concepts in this paper, we refer the reader to [37]. 
Let $Z$ be a metric space and $\pi: R^{+} \times Z \rightarrow Z$ a semi-flow (which is not necessarily continuous). For a given $M \in \mathbb{N}$, write $\pi^{(M)}=\underbrace{\pi \times \pi \times \cdots \times \pi}_{M}: R^{+} \times Z^{M} \rightarrow Z^{M}$. The semi-flow $\pi^{(M)}$ is called the M-power semi-flow of the semi-flow $\pi$. A point $z \in Z$ is said to be a transitive point of the semi-flow $\pi$, if the orbit $\mathrm{O}_{+}(z, \pi)$ of $z$ is dense in $Z$, where $\mathrm{O}_{+}(z, \pi)=\left\{\pi_{\mathrm{a}}(z): \mathrm{a} \in \mathrm{R}^{+}\right\}$. The semi-flow $\pi$ is said to be (topologically) transitive, if there exists a transitive point of the semi-flow $\pi$ in $Z$. A point $z \in Z$ is called a recurrent point of the semi-flow $\pi$, if there is an increasing sequence $\left\{r_{i}\right\} \subset R^{+}$with $\lim _{i \rightarrow \infty} r_{i}=\infty$ and $\lim _{i \rightarrow \infty} \varphi_{r_{i}}(z)=z$. Let $\operatorname{Rec}(\varphi)$ denote the set of all recurrent points of the semi-flow $\pi$.

Let $Z$ be a metric space. A subset $B \subset Z$ is said to be a $G_{\delta}$ set, if it is a countable intersection of open subset of $Z$. A subset $B \subset Z$ is said to be residual, if it contains a dense $G_{\delta}$ subset of $Z$.

In dynamical systems, sensitivity is a remarkable notion and property. Now we generalize it as follows.

Definition 2.1. Let $\pi: R^{+} \times Z \rightarrow Z$ (resp. $l: Z \rightarrow Z$ ) be a semi-flow (resp. a map) (which is not necessarily continuous) on a metric space $(Z, d)$. For a given integer $M \geqslant 2$, if there exists a $\lambda \in R^{+}$satisfying that for any nonempty open subset $\mathrm{V} \subset \mathrm{Z}$ there exist $z_{1}, z_{2}, \cdots, z_{M} \in Z$ with

$$
\min \left\{d\left(\pi_{s}\left(z_{\mathfrak{i}}\right), \pi_{s}\left(z_{j}\right)\right): i, j \in\{1,2, \cdots, M\} ; i \neq j\right\} \geqslant \lambda,
$$

for some $s \in R^{+}$(resp.

$$
\min \left\{d\left(l^{k}\left(z_{i}\right), l^{k}\left(z_{j}\right)\right): i, j \in\{1,2, \cdots, M\} ; i \neq j\right\} \geqslant \lambda,
$$

for some $k \geqslant 0$ ), then we say the number $\lambda$ is called an $M$-sensitive coefficient of the semi-flow $\pi$ (resp. the map $l$ or the system $(Z, l)$ ). The supremum of all $M$-sensitive coefficients of the semi-flow $\pi$ (resp. the map l) is said to be the $M$-critically sensitive coefficient of the semi-flow $\pi$ (resp. the map $l$ or the system $(Z, l))$ and is denoted by $\lambda_{M}(\pi)\left(\right.$ resp. $\left.\lambda_{M}(l)\right)$.

Let $\pi: R^{+} \times Z \rightarrow Z$ (resp. $l: Z \rightarrow Z$ ) be a semi-flow (resp. a map) (which is not necessarily continuous) on a metric space $(Z, d)$. For any integer $M \geqslant 2$ and any $p \in\{1,2, \cdots, M\}$, one can give three real-valued functions as follows.

$$
\phi_{M, p}\left(\pi,\left(z_{1}, z_{2}, \cdots, z_{M}\right)\right)=\sup _{t \geqslant 0} \min \left\{d\left(\pi_{t}\left(z_{p}\right), \pi_{t}\left(z_{q}\right)\right): q=1,2, \cdots, M ; q \neq p\right\},
$$

$\left(\right.$ resp. $\left.\phi_{M, p}\left(l,\left(z_{1}, z_{2}, \cdots, z_{M}\right)\right)=\sup _{m \geqslant 0} \min \left\{d\left(l^{m}\left(z_{p}\right), l^{m}\left(z_{q}\right)\right): q=1,2, \cdots, M ; q \neq p\right\}\right)$,

$$
\phi_{M}\left(\pi,\left(z_{1}, z_{2}, \cdots, z_{M}\right)\right)=\min _{1 \leqslant p \leqslant M} \phi_{M, p}\left(\pi,\left(z_{1}, z_{2}, \cdots, z_{M}\right)\right),
$$

$\left(\right.$ resp. $\left.\phi_{M}\left(l,\left(z_{1}, z_{2}, \cdots, z_{M}\right)\right)=\min _{1 \leqslant p \leqslant M} \phi_{M, p}\left(l,\left(z_{1}, z_{2}, \cdots, z_{M}\right)\right)\right)$ and

$$
\Phi_{M}\left(\pi,\left(z_{1}, z_{2}, \cdots, z_{M}\right)\right)=\min _{1 \leqslant p \leqslant M} \limsup _{t \rightarrow \infty} \min \left\{d\left(\pi_{t}\left(z_{p}\right), \pi_{t}\left(z_{q}\right)\right): q=1,2, \cdots, M ; q \neq p\right\},
$$

$\left(\right.$ resp. $\left.\Phi_{M}\left(l,\left(z_{1}, z_{2}, \cdots, z_{M}\right)\right)=\min _{1 \leqslant p \leqslant M} \limsup _{m \rightarrow \infty} \min \left\{d\left(l^{m}\left(z_{p}\right), l^{m}\left(z_{q}\right)\right): q=1,2, \cdots, M ; q \neq p\right\}\right)$.

Throughout this article, we always assume that $\pi$ is a continuous semi-flow on a complete metric space.

\section{Main results}

In [37] the author established that for any transitive system $(Z, l)$, the set of all transitive points of $l$ is a dense $G_{\delta}$ set. However, for continuous semi-flows one can obtain the following lemma. 
Lemma 3.1. For a continuous semi-flow $\pi: \mathrm{R}^{+} \times \mathbf{Z} \rightarrow \mathrm{Z}$ on a compact metric space $\mathrm{Z}$ with a countable base, the set of all transitive points of $\pi$ is a dense $\mathrm{G}_{\delta}$ set.

Proof. Let $\left\{V_{j}\right\}_{j=1}^{\infty}$ be a countable base of $Z$ and $\operatorname{Tr}(\pi)$ be the set of all transitive points of $\pi$. Then one has that

$$
\operatorname{Tr}(\pi)=\bigcap_{j \geqslant 1}\left(\bigcup_{t \geqslant 0} \pi_{t}^{-1}\left(V_{j}\right)\right)
$$

This implies that the set of all transitive points of $\pi$ is a dense $G_{\delta}$ set by the definition.

In [37], the author induced that for a transitive system $(Z, l)$ on a complete metric space $Z, \operatorname{Rec}\left(l^{(M)}\right)$ is a dense $G_{\delta}$ set in $Z^{M}$ for each integer $M \geqslant 1$. The following lemma is the semi-flow version of this result.

Proposition 3.2. For a transitive and continuous semi-flow $\pi: R^{+} \times \mathbf{Z} \rightarrow \mathbf{Z}$ on a compact metric space $\mathbf{Z}$ and any integer $\mathrm{M} \geqslant 1, \operatorname{Rec}\left(\pi^{(\mathrm{M})}\right)$ is a dense $\mathrm{G}_{\delta}$ set in $\mathrm{Z}^{\mathrm{M}}$.

Proof. Let $z \in Z$ be a transitive point of $\pi$. Then the point $z$ is a recurrent point of $\pi$. So, there is a sequence $\left\{r_{i}\right\} \subset R^{+}$with $\lim _{i \rightarrow \infty} r_{i}=\infty$ and $\lim _{i \rightarrow \infty} \pi_{r_{i}}(z)=z$. Consequently, for any $s_{1}, s_{2}, \cdots, s_{M} \in R^{+}$one obtains that

$$
\begin{aligned}
\lim _{i \rightarrow \infty}\left(\pi^{(M)}\right)_{r_{i}}\left(\pi_{s_{1}}(z), \pi_{s_{2}}(z), \cdots, \pi_{s_{M}}(z)\right) \\
\quad=\lim _{i \rightarrow \infty}\left(\pi_{s_{1}}\left(\pi_{r_{i}}(z)\right), \pi_{s_{2}}\left(\pi_{r_{i}}(z)\right), \cdots, \pi_{s_{N}}\left(\pi_{r_{i}}(z)\right)\right)=\left(\pi_{s_{1}}(z), \pi_{s_{2}}(z), \cdots, \pi_{s_{M}}(z)\right) .
\end{aligned}
$$

Hence, $\left(\pi_{s_{1}}(z), \pi_{s_{2}}(z), \cdots, \pi_{s_{M}}(z)\right) \in \operatorname{Rec}\left(\pi^{(M)}\right)$. This means every point of $Z^{M}$ is a non-wandering point of $\pi^{(M)}$. By $[22,23]$, we know that $\operatorname{Rec}\left(\pi^{(M)}\right)$ is a dense $G_{\delta}$ set in $Z^{M}$ for any integer $M \geqslant 1$.

Proposition 3.3. Let $\pi: R^{+} \times Z \rightarrow Z$ be a transitive and continuous semi-flow on a complete metric space $Z$ such that $\pi_{\mathrm{s}}$ is transitive for some $s>0$ and $r_{0} \geqslant 0$ be the infimum of the diameters of all invariant sets of the semi-flow $\pi$. Then, for any integer $M \geqslant 1$ there exists a dense $\mathrm{G}_{\delta}$ set $\mathrm{L}_{M} \subset \mathrm{Z}^{\mathrm{M}}$ with

$$
\liminf _{t \rightarrow \infty} \max \left\{d\left(\pi_{t}\left(z_{m}\right), \pi_{t}\left(z_{n}\right)\right): m, n \in\{1,2, \cdots, M\}\right\} \leqslant r_{0},
$$

for any $\left(z_{1}, z_{2}, \cdots, z_{M}\right) \in \mathrm{L}_{M}$.

Proof. For the above $s>0$, we let $r_{0}^{\prime} \geqslant 0$ be the infimum of the diameters of all invariant sets of the map $\pi_{s}$. By [37, Proposition 3.5], for any integer $M \geqslant 1$ there exists a dense $G_{\delta}$ set $L_{M} \subset Z^{M}$ with

$$
\liminf _{k \rightarrow \infty} \max \left\{d\left(\pi_{s}^{k}\left(z_{m}\right), \pi_{s}^{k}\left(z_{n}\right)\right): m, n \in\{1,2, \cdots, M\}\right\} \leqslant r_{0}^{\prime}
$$

for any $\left(z_{1}, z_{2}, \cdots, z_{M}\right) \in \mathrm{L}_{M}$. Clearly, one has that

$$
\begin{aligned}
& \liminf _{\mathrm{t} \rightarrow \infty} \max \left\{\mathrm{d}\left(\pi_{\mathrm{t}}\left(z_{\mathrm{m}}\right), \pi_{\mathrm{t}}\left(z_{\mathrm{n}}\right)\right): \mathrm{m}, \mathrm{n} \in\{1,2, \cdots, M\}\right\} \\
& \quad \leqslant \liminf _{k \rightarrow \infty} \max \left\{\mathrm{d}\left(\pi_{\mathrm{s}}^{\mathrm{k}}\left(z_{\mathrm{m}}\right), \pi_{\mathrm{s}}^{\mathrm{k}}\left(z_{\mathrm{n}}\right)\right): \mathrm{m}, \mathrm{n} \in\{1,2, \cdots, M\}\right\} .
\end{aligned}
$$

Since $r_{0}^{\prime} \leqslant r_{0}$

$$
\liminf _{t \rightarrow \infty} \max \left\{d\left(\pi_{t}\left(z_{m}\right), \pi_{t}\left(z_{n}\right)\right): m, n \in\{1,2, \cdots, M\}\right\} \leqslant r_{0},
$$

for any $\left(z_{1}, z_{2}, \cdots, z_{M}\right) \in \mathrm{L}_{M}$.

Remark 3.4. For a transitive continuous semi-flow $\pi$, we do not know whether the conclusion of Proposition 3.3 holds. 
Proposition 3.5. Let $\pi: \mathrm{R}^{+} \times \mathrm{Z} \rightarrow \mathrm{Z}$ be a semi-flow on a locally connected metric space $\mathrm{Z}$. Then, for any integer $M \geqslant 2$ one has that

$$
\lambda_{M}(\pi) \geqslant \frac{\lambda_{2}(\pi)}{M-1} .
$$

Proof. Assume that $\beta$ is a base of $Z$ such that each member in $\beta$ is connected. By the definition, for any $V \in \beta$ there exist two points $u, v \in V$ with $d\left(\pi_{s}(u), \pi_{s}(v)\right) \geqslant \lambda_{2}(\pi)$ for some $s \geqslant 0$. Let $h: V \rightarrow \mathbb{R}$ be a function which is defined by $h(w)=d\left(\pi_{s}(w), \pi_{s}(u)\right)$ for any $w \in V$. As $V$ is connected, for any integer $M \geqslant 2$ there exist $M$ points $u=u_{1}, u_{2}, \cdots, u_{M}=v \in V$ with $h\left(u_{m}\right)=\frac{m-1}{M-1} d\left(\pi_{s}(v), \pi_{s}(u)\right)$ for all $1 \leqslant m \leqslant M$. For any $m, n \in\{1,2, \cdots, M\}$ with $m<n$, one can get

$$
d\left(\pi_{s}\left(u_{1}\right), \pi_{s}\left(u_{n}\right)\right) \leqslant d\left(\pi_{s}\left(u_{1}\right), \pi_{s}\left(u_{m}\right)\right)+d\left(\pi_{s}\left(u_{m}\right), \pi_{s}\left(u_{n}\right)\right) .
$$

Therefore, for any $m, n \in\{1,2, \cdots, M\}$ with $m<n$, we get

$$
d\left(\pi_{s}\left(u_{m}\right), \pi_{s}\left(u_{n}\right)\right) \geqslant \frac{n-m}{M-1} d\left(\pi_{s}(u), \pi_{s}(v)\right) \geqslant \frac{1}{M-1} d\left(\pi_{s}(u), \pi_{s}(v)\right) \geqslant \frac{1}{M-1} \lambda_{2}(\pi) .
$$

This means that $\lambda_{m}(\pi) \geqslant \frac{1}{M-1} \lambda_{2}(\pi)$.

Proposition 3.6. Let $\pi: R^{+} \times Z \rightarrow Z$ be a continuous semi-flow on a complete metric space $Z$ such that $\pi_{\mathrm{s}}$ is transitive for some $s>0$ and let $\lambda \geqslant 0$ be an $M$-sensitive coefficient of the map $\pi_{s}$, where $M \geqslant 2$. Then, the set consisting of the points $\left(\mathrm{u}_{1}, \mathrm{u}_{2}, \cdots, \mathrm{u}_{M}\right)$ with

$$
\Phi_{M}\left(\pi,\left(u_{1}, u_{2}, \cdots, u_{M}\right)\right) \geqslant \frac{\lambda}{2}
$$

is a residual set in the product space $\mathrm{Z}^{\mathrm{M}}$.

Proof. By [37, Proposition 5.2], the set consisting of the points $\left(u_{1}, u_{2}, \cdots, u_{M}\right)$ with

$$
\Phi_{M}\left(\pi_{s},\left(u_{1}, u_{2}, \cdots, u_{M}\right)\right) \geqslant \frac{\lambda}{2}
$$

is a residual set in the product space $Z^{M}$ for any integer $M \geqslant 2$, where $\lambda$ is an $M$-sensitive coefficient of the map $\pi_{s}$. By the definition,

$$
\Phi_{M}\left(\pi,\left(u_{1}, u_{2}, \cdots, u_{M}\right)\right) \geqslant \Phi_{M}\left(\pi_{s},\left(u_{1}, u_{2}, \cdots, u_{M}\right)\right) .
$$

So, the set consisting of the points $\left(u_{1}, u_{2}, \cdots, u_{M}\right)$ with

$$
\Phi_{M}\left(\pi,\left(u_{1}, u_{2}, \cdots, u_{M}\right)\right) \geqslant \frac{\lambda}{2}
$$

is a residual set in the product space $Z^{M}$.

Remark 3.7. For a transitive continuous semi-flow $\pi$, we do not know whether the conclusion of Proposition 3.6 holds.

Lemma 3.8. Let $\pi: R^{+} \times Z \rightarrow Z$ be a continuous semi-flow on a complete metric space $\mathbf{Z}$ such that $\pi_{\mathrm{s}}$ is topologically transitive for some $s \in R^{+}$and $w \in Z$ be a transitive point of the map $\pi_{s}$, and let $M \geqslant 2$ be an integer and $j \in\{1,2, \cdots, M\}$. If $\lambda \geqslant 0$ is an $M$-sensitive coefficient of the map $\pi_{s}$, then for any integers $k_{1}, k_{2}, \cdots, k_{M} \geqslant 1$ and any neighborhood $W$ of the point $\left(\pi_{\mathrm{s}}^{k_{1}}(w), \pi_{\mathrm{s}}^{k_{2}}(w), \cdots, \pi_{\mathrm{s}}^{k_{M}}(w)\right)$, there exists a point $\left(\mathrm{u}_{1}, \mathrm{u}_{2}, \cdots, \mathrm{u}_{M}\right) \in W$ with

$$
\phi_{M, j}\left(\pi,\left(u_{1}, u_{2}, \cdots, u_{M}\right)\right) \geqslant \frac{\lambda}{2}
$$


Proof. From [37, Lemma 5.1] we know that for any integers $k_{1}, k_{2}, \cdots, k_{M}$ and any neighborhood $W$ of the point $\left(\pi_{\mathrm{s}}^{\mathrm{k}_{1}}(w), \pi_{\mathrm{s}}^{k_{2}}(w), \cdots, \pi_{\mathrm{s}}^{k_{M}}(w)\right)$, there exists a point $\left(\mathrm{u}_{1}, \mathrm{u}_{2}, \cdots, u_{M}\right) \in W$ with

$$
\phi_{M, j}\left(\pi_{s},\left(u_{1}, u_{2}, \cdots, u_{M}\right)\right) \geqslant \frac{\lambda}{2} \text {. }
$$

As

$$
\phi_{M, j}\left(\pi,\left(u_{1}, u_{2}, \cdots, u_{M}\right)\right) \geqslant \phi_{M, j}\left(\pi_{s},\left(u_{1}, u_{2}, \cdots, u_{M}\right)\right),
$$

for the above integers $k_{1}, k_{2}, \cdots, k_{N}$ and the above neighborhood $W$ of the point

$$
\left(\pi_{\mathrm{s}}^{\mathrm{k}_{1}}(w), \pi_{\mathrm{s}}^{\mathrm{k}_{2}}(w), \cdots, \pi_{\mathrm{s}}^{\mathrm{k}_{M}}(w)\right),
$$

there exists a point $\left(\mathfrak{u}_{1}, \mathfrak{u}_{2}, \cdots, u_{M}\right) \in W$ with

$$
\phi_{M, j}\left(\pi,\left(u_{1}, u_{2}, \cdots, u_{M}\right)\right) \geqslant \frac{\lambda}{2}
$$

Remark 3.9. For a transitive continuous semi-flow $\pi$, we do not know whether the conclusion of Lemma 3.8 holds.

Theorem 3.10. Let $\pi: R^{+} \times Z \rightarrow Z$ be a continuous semi-flow on a complete metric space $\mathrm{Z}$ with a metric $\mathrm{d}$ such that $\mathrm{Z}$ is dense in itself, and that $\pi_{\mathrm{s}}$ is topologically transitive for some $\mathrm{s} \in \mathrm{R}^{+}$. Then there exists a subset $\mathrm{K} \subset \mathrm{Z}$ such that $\mathrm{K} \cap \mathrm{V}$ contains a nonempty compact perfect set for any nonempty open subset $\mathrm{V} \subset \mathrm{Z}$. Moreover, the following are true:

(1) each point in $\mathrm{K}$ is transitive;

(2) for any integer $\mathrm{M} \geqslant 2$ and any pairwise different $M$ points $\mathrm{u}_{1}, \mathrm{u}_{2}, \cdots, \mathrm{u}_{\mathrm{M}}$ in $\mathrm{K}$, we have

$$
\limsup _{t \rightarrow \infty} \min \left\{d\left(\pi_{t}\left(u_{m}\right), \pi_{t}\left(u_{n}\right)\right): m, n \in\{1,2, \cdots, M\} ; m \neq n\right\}>0
$$

(3) for any integer $M \geqslant 2$ and any pairwise different $M$ points $u_{1}, u_{2}, \cdots, u_{M}$ in $K$, we have

$$
\liminf _{t \rightarrow \infty} \max \left\{d\left(\pi_{t}\left(u_{m}\right), \pi_{t}\left(u_{n}\right)\right): m, n \in\{1,2, \cdots, M\}\right\} \leqslant r_{0},
$$

where $r_{0}$ is the infimum of the diameters of all invariant sets of the map $\pi_{\mathrm{s}}$;

(4) for any integer $M \geqslant 2$ and any pairwise different $M$ points $u_{1}, u_{2}, \cdots, u_{M}$ in $K$,

$$
\min _{1 \leqslant i \leqslant N} \limsup _{t \rightarrow \infty} \min \left\{d\left(\pi_{t}\left(u_{m}\right), \pi_{t}\left(u_{n}\right)\right): n \in\{1,2, \cdots, M\} ; n \neq m\right\} \geqslant \frac{\lambda_{M}\left(\pi_{s}\right)}{2},
$$

where $\lambda_{M}\left(\pi_{s}\right)$ is the $M$-critically sensitive coefficient of the map $\pi_{s}$; and if the space $Z$ is locally connected then for any integer $M \geqslant 2$ and any pairwise different $M$ points $u_{1}, u_{2}, \cdots, u_{M}$ in $K$,

$$
\min _{1 \leqslant j \leqslant M} \limsup _{t \rightarrow \infty} \min \left\{d\left(\pi_{t}\left(u_{m}\right), \pi_{t}\left(u_{n}\right)\right): n \in\{1,2, \cdots, M\} ; n \neq m\right\} \geqslant \frac{\lambda_{2}(\pi)}{2 M-2} .
$$

Proof. It is noted that for any $v_{1}, v_{2}, \cdots, v_{M} \in Z$, we have

$$
\begin{aligned}
& \limsup _{\mathrm{t} \rightarrow \infty} \min \left\{\mathrm{d}\left(\pi_{\mathrm{t}}\left(v_{\mathrm{m}}\right), \pi_{\mathrm{t}}\left(v_{\mathrm{n}}\right)\right): \mathrm{m}, \mathrm{n} \in\{1,2, \cdots, M\} ; \mathrm{m} \neq \mathrm{n}\right\} \\
& \geqslant \limsup _{p \rightarrow \infty} \min \left\{d\left(\pi_{s}^{p}\left(v_{m}\right), \pi_{s}^{p}\left(v_{n}\right)\right): m, n \in\{1,2, \cdots, M\} ; m \neq n\right\}, \\
& \liminf _{t \rightarrow \infty} \max \left\{d\left(\pi_{t}\left(v_{m}\right), \pi_{t}\left(v_{n}\right)\right): m, n \in\{1,2, \cdots, M\}\right\} \\
& \leqslant \liminf _{p \rightarrow \infty} \max \left\{\mathrm{d}\left(\pi_{\mathrm{s}}^{\mathrm{p}}\left(v_{\mathrm{m}}\right), \pi_{\mathrm{s}}^{\mathrm{p}}\left(v_{\mathrm{n}}\right)\right): \mathrm{m}, \mathrm{n} \in\{1,2, \cdots, M\}\right\},
\end{aligned}
$$


and

$$
\begin{aligned}
\min _{1 \leqslant m \leqslant M} \limsup _{\mathrm{t} \rightarrow \infty} \min & \left\{\mathrm{d}\left(\pi_{\mathrm{t}}\left(v_{\mathrm{m}}\right), \pi_{\mathrm{t}}\left(v_{\mathrm{n}}\right)\right): \mathrm{n} \in\{1,2, \cdots, M\} ; \mathrm{n} \neq \mathrm{m}\right\} \\
& \geqslant \min _{1 \leqslant \mathrm{~m} \leqslant M} \limsup _{\mathrm{p} \rightarrow \infty} \min \left\{\mathrm{d}\left(\pi_{\mathrm{s}}^{\mathrm{p}}\left(v_{\mathrm{m}}\right), \pi_{\mathrm{s}}^{\mathrm{p}}\left(v_{\mathrm{n}}\right)\right): \mathrm{n} \in\{1,2, \cdots, M\} ; \mathrm{n} \neq \mathrm{m}\right\} .
\end{aligned}
$$

By Theorem in [37] and Proposition 3.5, the results of Theorem 3.10 are true.

Remark 3.11. For a transitive continuous semi-flow $\pi$, we do not know whether the conclusion of Theorem 3.10 holds.

\section{Acknowledgment}

The authors are very grateful to the referees for their careful reading, comments, and suggestions, which helped us to improve this paper.

This research was supported by the Project of Enhancing School With Innovation of Guangdong Ocean University (Grant NO. GDOU2016050207), the Key Scientific and Technological Research Project of Science and Technology Department of Zhanjiang City (Grant 2010C3112005) and the National Natural Science Foundation of China (11501391).

\section{References}

[1] C. Abraham, G. Biau, B. Cadre, On Lyapunov exponent and sensitivity for maps of the interval, Tech. Rep., Univ. Montpellier II, (2001). 1

[2] C. Abraham, G. Biau, B. Cadre, Chaotic properties of mappings on a probability space, J. Math. Anal. Appl., 266 (2002), 420-431. 1

[3] A. Arbieto, R. Ribeiro, Flows with the (asymptotic) average shadowing property on three-dimensional closed manifolds, Dyn. Syst., 26 (2011), 425-432. 1

[4] J. Banks, J. Brooks, G. Cairns, G. Davis, P. Stacey, On Devaney's definition of chaos, Amer. Math. Monthly, 99 (1992), 332-334. 1

[5] W. Basener, M. C. Sullivan, Periodic prime knots and topologically transitive flows on 3-manifolds, Topology Appl., 153 (2006), 1236-1240. 1

[6] E. Bilokopytov, S. F. Kolyada, Transitive maps on topological spaces, Ukrainian Math. J., 65 (2014), 1293-1318. 1

[7] R. L. Devaney, An introduction to chaotic dynamical systems, Second edition, Addison-Wesley Studies in Nonlinearity, Addison-Wesley Publishing Company, Advanced Book Program, Redwood City, CA, (1989). 1

[8] R.-B. Gu, The asymptotic average-shadowing property and transitivity for flows, Chaos Solitons Fractals, 41 (2009), 2234-2240. 1

[9] R.-B. Gu, W.-J. Guo, The average-shadowing property and topological ergodicity for flows, Chaos Solitons Fractals, 25 (2005), 387-392. 1

[10] R.-B. Gu, Z.-J. Xia, The average-shadowing property and transitivity for continuous flows, Chaos Solitons Fractals, 23 (2005), 989-995. 1

[11] L.-F. He, Y.-H. Gao, F.-H. Yang, Some dynamical properties of continuous semi-flows having topological transitivity, Chaos Solitons Fractals, 14 (2002), 1159-1167. 1

[12] L. Huang, D. Baleanu, G.-C. Wu, S.-D. Zeng, A new application of the fractional logistic map, Rom. J. Phys., 61 (2016), 1172-1179. 1

[13] W. Huang, X.-D. Ye, Devaney's chaos or 2-scattering implies Li-Yorke's chaos, Topology Appl., 117 (2002), 259-272. 1

[14] R.-S. Li, A note on decay of correlation implies chaos in the sense of Devaney, Appl. Math. Model., 39 (2015), 6705-6710. 1

[15] R.-S. Li, Y.-M. Shi, Stronger forms of sensitivity for measure-preserving maps and semiflows on probability spaces, Abstr. Appl. Anal., 2014 (2014), 10 pages. 1

[16] Y. T. Li, J. A. Yorke, Period three implies chaos, Amer. Math. Monthly, 82 (1975), 985-992. 1

[17] R.-S. Li, X.-L. Zhou, A note on ergodicity of systems with the asymptotic average shadowing property, Discrete Dyn. Nat. Soc., 2011 (2011), 6 pages. 1

[18] R.-S. Li, X.-L. Zhou, A note on chaos in product maps, Turkish J. Math., 37 (2013), 665-675.

[19] M. Magdziarz, A. Weron, Ergodic properties of anomalous diffusion processes, Ann. Physics, 326 (2011), 2431-2443. 1

[20] J.-H. Mai, Devaney's chaos implies existence of s-scrambled sets, Proc. Amer. Math. Soc., 132 (2004), 2761-2767. 1

[21] T. K. S. Moothathu, Stronger forms of sensitivity for dynamical systems, Nonlinearity, 20 (2007), 2115-2126. 1

[22] V. V. Nemytskii, V. V. Stepanov, Qualitative theory of differential equations, Princeton Mathematical Series, Princeton University Press, Princeton, N.J., (1960). 3 
[23] V. V. Nemytskii, V. V. Stepanov, Qualitative theory of differential equations, Dover, New York, (1989). 3

[24] D. Ruelle, F. Takens, On the nature of turbulence, Comm. Math. Phys., 20 (1971), 167-192. 1

[25] X.-X. Wu, Chaos of transformations induced onto the space of probability measures, Internat. J. Bifur. Chaos Appl. Sci. Engrg., 26 (2016), 12 pages. 1

[26] X.-X. Wu, A remark on topological sequence entropy, Internat. J. Bifur. Chaos Appl. Sci. Engrg., (Accepted). 1

[27] G.-C. Wu, D. Baleanu, Chaos synchronization of the discrete fractional logistic map, Signal Process., 102 (2014), 96-99. 1

[28] G.-C. Wu, D. Baleanu, Discrete fractional logistic map and its chaos, Nonlinear Dynam., 75 (2014), 283-287. 1

[29] G.-C. Wu, D. Baleanu, H.-P. Xie, F.-L. Chen, Chaos synchronization of fractional chaotic maps based on the stability condition, Phys. A, 460 (2016), 374-383. 1

[30] X.-X. Wu, G.-R. Chen, Sensitivity and transitivity of fuzzified dynamical systems, Inform. Sci., 396 (2017), 14-23. 1

[31] X.-X. Wu, P. Oprocha, G.-R. Chen, On various definitions of shadowing with average error in tracing, Nonlinearity, 29 (2016), 1942-1972.

[32] X.-X. Wu, X. Wang, On the iteration properties of large deviations theorem, Internat. J. Bifur. Chaos Appl. Sci. Engrg., 26 (2016), 6 pages.

[33] X.-X. Wu, J.-J. Wang, G.-R. Chen, F-sensitivity and multi-sensitivity of hyperspatial dynamical systems, J. Math. Anal. Appl., 429 (2015), 16-26.

[34] X.-X. Wu, L.-D. Wang, G.-R. Chen, Weighted backward shift operators with invariant distributionally scrambled subsets, Ann. Funct. Anal., 8 (2017), 199-210.

[35] X.-X. Wu, L.-D. Wang, J.-H. Liang, The chain properties and average shadowing property of iterated function systems, Qual. Theory Dyn. Syst., 2016 (2016), 9 pages.

[36] X.-X. Wu, L.-D. Wang, J.-H. Liang, The chain properties and Li-Yorke sensitivity of zadeh's extension on the space of upper semi-continuous fuzzy sets, Iran. J. Fuzzy Syst., (Accepted). 1

[37] J.-C. Xiong, Chaos in a topologically transitive system, Sci. China Ser. A, 48 (2005), 929-939. 1, 2, 3, 3, 3, 3, 3, 3

[38] J.-C. Xiong, E. Chen, Chaos caused by a strong-mixing measure-preserving transformation, Sci. China Ser. A, 40 (1997), 253-260. 1

[39] J.-C. Xiong, Z. G. Yang, Chaos caused by a topologically mixing map, Dynamical systems and related topics, Nagoya, (1990), Adv. Ser. Dynam. Systems, World Sci. Publ., River Edge, NJ, 9 (1991), 550-572. 1

[40] J.-D. Yin, Z.-L. Zhou, A characterization of topologically transitive attributes for a class of dynamical systems, Chin. Ann. Math. Ser. B, 33 (2012), 419-428. 1 